\title{
Chemo Immunostimulant Adjuvant
}

National Cancer Institute

\section{Source}

National Cancer Institute. Chemo Immunostimulant Adjuvant. NCI Thesaurus. Code C2141.

Adjuvant used in chemotherapy that acts by stimulating the immune system. 